\title{
Extensión del tratamiento quirúrgico y quimioterapia adyuvante en pacientes con carcinoma incidental de la vesícula biliar
}

\author{
Extension of surgical treatment and adjuvant chemotherapy in patients with incidental \\ gallbladder cancer
}

\begin{abstract}
Carlos Manterola ${ }^{1,2 *}$, Luis Grande ${ }^{3,4,5}$, Tamara Otzen ${ }^{2,6}$ y Roque Conejeros ${ }^{7}$
${ }^{1}$ Departamento de Cirugía, Universidad de La Frontera, Temuco, Chile; ${ }^{2}$ Centro de Estudios Morfológicos y Quirúrgicos, Universidad de La Frontera, Temuco, Chile; ${ }^{3}$ Departamento de Cirugía, Universidad Autónoma de Barcelona, Barcelona, España; ${ }^{4}$ Servicio de Cirugía, Hospital Universitario del Mar, Barcelona, España; Institut Hospital del Mar d'Investigacions Mèdiques, Barcelona, España; ${ }^{6}$ Facultad de Ciencias de la Salud, Universidad de Tarapacá, Arica, Chile; ${ }^{7}$ Instituto de Oncología Clínica del Sur, Temuco, Chile
\end{abstract}

\section{Resumen}

Objetivo: Describir los resultados de la extensión del tratamiento quirúrgico y la quimioterapia adyuvante en el cáncer de vesícula biliar (CVB) incidental, en términos de morbilidad posoperatoria (MPO) y de supervivencia global (SVG) a 5 años. Método: Serie de casos de pacientes intervenidos por CVB incidental en Clínica Mayor, Temuco, Chile (2001-2016). Todos los casos fueron tratados mediante hepatectomía parcial de segmentos IVb y V, y linfadenectomía regional. El tiempo mínimo de seguimiento fue de 12 meses. La variables resultado fueron: MPO y la SVG a 5 años. Otras variables de interés fueron: profundidad de infiltración en la pared vesicular, de linfonodos y del hígado resecado, tiempo quirúrgico, necesidad de reintervención, estancia hospitalaria, seguimiento y mortalidad. Se aplicó estadística descriptiva, y análisis bivariados, aplicando prueba exacta de Fisher y t-test; o pruebas no paramétricas para variables continuas; y curvas de Kaplan-Meier. Resultados: La serie está compuesta por 50 pacientes, con edad media de $58.6 \pm 9.6$ años; $68.0 \%$ de los cuales eran mujeres. La media del tiempo quirúrgico y de estancia hospitalaria fueron $224 \pm 93$ minutos (90 a 480) y 6.9 2.9 días (4 a 20) respectivamente. La MPO fue 28.0\%. La SVG a 5 años fue $47 \%$. No hubo reintervenciones ni mortalidad. Conclusiones: Los resultados verificados en términos de MPO y SVG a 5 años, son similares a los reportados anteriormente.

PALABRAS CLAVE: Cáncer de la vesicular biliar. Hepatectomía. Linfadenectomía. Quimioterapia adyuvante. Colecistectomía laparoscópica. Kaplan-Meier.

\begin{abstract}
Objective: To describe the results of the extension of surgical treatment and adjuvant chemotherapy (ACT) in incidental gallbladder cancer (CVB), in terms of postoperative morbidity (POM) and 5-year overall survival rate. Method: Case series of patients operated on for incidental GBC in Clínica Mayor, Temuco, Chile (2001-2016). All cases were treated by partial hepatectomy (segments IVb and V), and regional lymphadenectomy. The minimum follow-up time was 12 months. Outcome variables were MPO and 5-year overall survival rate. Other variables of interest were: infiltration depth in vesicular wall, lymph nodes and resected liver; surgical time, need for reoperation, hospital stay, follow-up and mortality. Descriptive statistics were applied as well as bivariate analysis applying Fisher's exact and t-test and non-parametrical tests for continuous variables and Kaplan Meier curves. Results: The series was composed of 50 patients, whose average age was $58.6 \pm 9.6$ years; $68.0 \%$ of which were women. The mean surgical time and hospital stay were $224 \pm 93$ min (90 to 480$)$ and $6.9 \pm$ 2.9 days (4 to 20), respectively. POM was $28.0 \%$. 5-year overall survival rate was $47 \%$. There were no reoperations or
\end{abstract}

\author{
Correspondencia: \\ ${ }^{*}$ Carlos Manterola \\ Manuel Montt 112, Oficina 408, Casilla 54-D \\ C.P. 4780000 , Temuco, Chile \\ E-mail: carlos.manterola@ufrontera.cl
}

Fecha de recepción: 12-07-2018

Fecha de aceptación: 15-12-2018

DOI: 10.24875/CIRU.18000596
Cir Cir. 2019;87:313-320

Contents available at PubMed www.cirugiaycirujanos.com 
mortality. Conclusions: The results verified in terms of POM and 5-year overall survival rate are similar to previously reported series.

KEY WORDS: Gallbladder cancer. Hepatectomy. Lymphadenectomy. Chemotherapy Adjuvant. Laparoscopic cholecystectomy. Kaplan-Meier.

\section{Introducción}

El cáncer de vesícula biliar (CVB) representa la segunda causa de muerte por cáncer en las mujeres en Chile, después del cáncer de mama ${ }^{1}$. La supervivencia a 5 años está en relación con el estadio patológico (I, 50\%; II, 28\%; III, 8\%; y IV, 2\%) 2, $^{2,3}$

La mayoría de los pacientes que sobreviven a largo plazo son aquellos a quienes se diagnosticó una neoplasia incidental en el estudio patológico de una colecistectomía electiva o de urgencias ${ }^{4}$, pero desgraciadamente el diagnóstico suele realizarse en estadios avanzados 5 . Se dispone de una serie de factores que apuntan al mal pronóstico, como la localización de la neoplasia en la cara hepática de la vesícula biliar ${ }^{6,7}$, el grado de infiltración de la pared vesicular, el grado de diferenciación tumoral, la infiltración perineural o vascular ${ }^{5,8,9}$, el compromiso de los senos de Rokitansky-Aschoff ${ }^{10}$ y la existencia y la extensión de la infiltración linfonodal $\left.\right|^{8,11-13}$.

Por lo que respecta al tratamiento, existe evidencia de que en el CVB con invasión limitada a la capa mucosa ( $\mathrm{pT} 1 \mathrm{a})$ sería suficiente la colecistectomía. En los casos de invasión en la capa muscular (pT1b) no hay consenso respecto a la utilidad del tratamiento complementario (quirúrgico, quimioterapia o radioterapia), que parece imponerse en los casos en que la invasión alcanza la subserosa (pT2)3,9,11,14-16. En los pacientes en quienes se detecte compromiso de la serosa (pT3) o del tejido adiposo seroso o perivesicular (pT4), el uso de tratamientos complementarios es discutible porque, en estos casos, suele encontrarse extensión tumoral a órganos adyacentes y metástasis hepáticas durante la cirugía, lo que hace que cualquier tratamiento adicional sea poco eficiente ${ }^{3,9,17}$.

De acuerdo con estas evidencias, se han utilizado diferentes protocolos terapéuticos para el CVB, pero en general se acepta la realización de una resección hepática, asociada o no a protocolos adyuvantes o neoadyuvantes ${ }^{3,14,15,18-20}$, como tratamiento de elección.

El objetivo de este estudio fue describir los resultados de la extensión del tratamiento quirúrgico y la quimioterapia adyuvante en el CVB incidental, diagnosticado en la pieza quirúrgica de una colecistectomía, en términos de morbilidad posoperatoria (MPO) y de supervivencia global (SVG) a 5 años.

\section{Método}

El reporte de este estudio fue escrito siguiendo la propuesta MInCir para el reporte de estudios observacionales descriptivos ${ }^{21}$.

\section{Diseño}

Serie de casos con seguimiento.

\section{Escenario}

El estudio se realizó en Clínica Mayor, Temuco, Chile. El periodo de reclutamiento fue entre enero de 2001 y diciembre de 2016 (16 años).

\section{Participantes}

En esta serie se incluyen solo aquellos pacientes en los que, tras una colecistectomía laparoscópica por colelitiasis, se confirmó histológicamente la existencia de un CVB en estadio pT1b o superior.

\section{Tamaño de muestra}

Se trabajó con la totalidad de los casos asistidos, sin exclusión alguna.

\section{Variables en estudio}

Las variables principales fueron el desarrollo de MPO y la SVG a 5 años. Otras variables de interés fueron la edad, el sexo, los datos de laboratorio, la profundidad de infiltración en la pared vesicular, linfonodal y hepática, el tiempo quirúrgico, la necesidad de reintervención, la estancia hospitalaria, el tiempo de seguimiento y la mortalidad relacionada con el tratamiento. La variable MPO fue medida de forma 


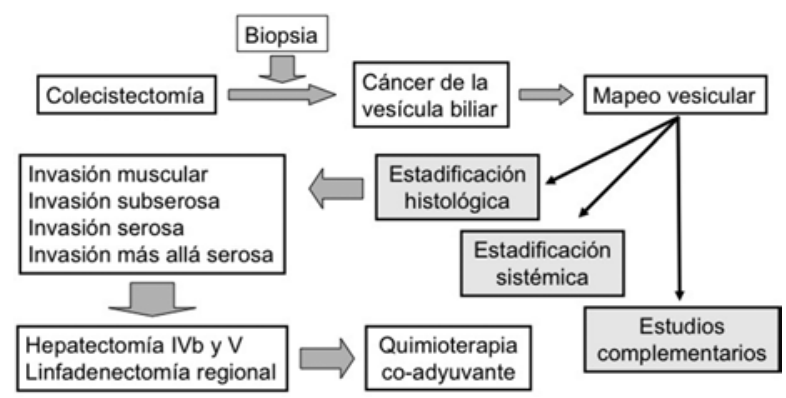

Figura 1. Algoritmo diagnóstico-terapéutico utilizado en los pacientes de esta serie.

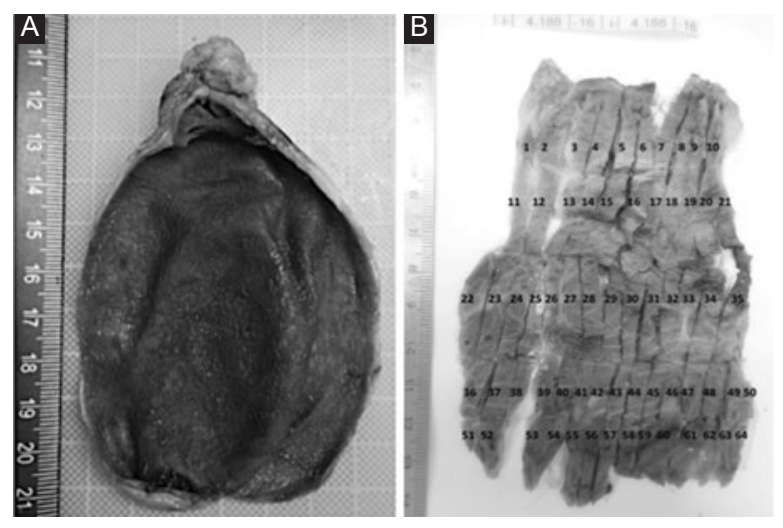

Figura 2. Espécimen de colecistectomía fijado en formalina (A) y posteriormente mapeado en pequeños fragmentos para estudio independiente (B), con la finalidad de determinar el mayor nivel de invasión tumoral en la pared vesicular.

dicotómica (presente/ausente) utilizando la propuesta de Clavien-Dindo²2.

\section{Protocolo de estudio}

El protocolo aplicado se describe en la figura 1. De forma sucinta, una vez confirmada la existencia de una neoplasia en el espécimen de la vesícula biliar (estudio básico, con análisis de tres muestras: fondo, cuerpo y cuello), se realizó un «mapeo» para determinar el nivel de mayor profundidad de infiltración en la pared vesicular (Fig. 2). Los pacientes pT1a se consideraron «curados» con la colecistectomía, por lo que no se realizó ninguna actuación más, razón por la cual no fueron incluidos en este estudio. En los casos de invasión pT1b, pT2, pT3 y pT4 se realizó la resección de los segmentos hepáticos IVb y V $(s 4 b+$ s5), asociada a linfadenectomía $D_{2}$ (ganglios interaortocava, del área coledocoduodenal, retropancreáticos, del pedículo hepático y del tronco celíaco ${ }^{23,24}$. También se agregó la resección de cicatrices quirúrgicas de los puertos laparoscópicos ${ }^{9}$. En los casos pT4 con invasión evidente del parénquima hepático o de la vía biliar, se realizaron resecciones mayores (triseccionectomía derecha o hepatectomía derecha), según necesidades. Tras la colecistectomía, a todos los casos se les practicó una tomografía computada de estadificación, previamente a la cirugía de resección para el tratamiento de la neoplasia. Después de la segunda cirugía se administró quimioterapia durante 6 meses con gemcitabina y 5 -fluorouracilo (5-FU), cuyo primer ciclo comenzó en la cuarta semana del posoperatorio.

\section{Procedimiento quirúrgico}

Se utilizó laparotomía en $\mathrm{J}$ en todos los casos. Se realizó exploración manual seguida de movilización total del hígado, y se seccionaron los ligamentos suspensorio, triangulares y coronales. Además, se realizó ecografía intraoperatoria para determinar la existencia de eventuales metástasis y la distribución del sistema venoso portal y de las venas hepáticas. Posteriormente se practicó una linfadenectomía $\mathrm{D}_{2}$. La transección hepática se realizó bajo oclusión del pedículo hepático con una pinza de Rumel con electrocoagulación monopolar y Ligasure $^{\circledR}$. Por último, se movilizó un parche pediculado de epiplón mayor al área cruenta, donde se instaló un drenaje de silicona que se exteriorizó por contraabertura.

\section{Protocolo de seguimiento}

Tras el alta hospitalaria se realizaron controles clínicos, de laboratorio y radiológicos a todos pacientes a los 1,3 y 6 meses de la intervención, y luego al menos una vez al año.

\section{Análisis estadísticos}

La recopilación de datos fue realizada por un investigador independiente (TO) y analizada con el programa Stata 11.0/SE ${ }^{\circledR}$. Se utilizaron estadísticas descriptivas, aplicando cálculo de porcentajes y medidas de tendencia central y dispersión (media y desviación estándar, mediana y valores extremos). Además, se estimó la supervivencia con curvas de KaplanMeier. Finalmente, se aplicó estadística bivariada utilizando la prueba de Fisher para variables categóricas y la prueba t de Student para variables continuas.

\section{Sesgos}

Se enmascaró la recopilación de datos y se minimizaron los sesgos mediante el seguimiento completo 
Tabla 1. Variables clínicas y biodemográficas de los pacientes incluidos en el estudio $(n=50)$

\begin{tabular}{|c|c|c|c|c|c|}
\hline \multirow[t]{2}{*}{ Variable } & \multicolumn{5}{|c|}{ Nivel de infiltración tumoral } \\
\hline & $\begin{array}{c}\text { pT1b } \\
(n=11)\end{array}$ & $\begin{array}{c}\text { pT2 } \\
(n=13)\end{array}$ & $\begin{array}{c}\text { pT3 } \\
(n=13)\end{array}$ & $\begin{array}{c}\text { pT4 } \\
(n=13)\end{array}$ & $\mathbf{p}$ \\
\hline Edad (años) ${ }^{*}$ & $56.2 \pm 12.4$ & $60.8 \pm 5.8$ & $61.0 \pm 7.9$ & $55.9 \pm 11.4$ & 0.058 \\
\hline Sexo femenino, n (\%) & $10(90.9)$ & $9(69.2)$ & $10(76.9)$ & $3(38.5)$ & 0.048 \\
\hline Infiltración del hígado, n (\%) & $0(0.0)$ & $2(15.4)$ & $6(46.2)$ & $13(100.0)$ & 0.001 \\
\hline Infiltración linfonodal, n (\%) & $3(27.3)$ & $7(53.8)$ & $11(84.6)$ & $13(100.0)$ & 0.001 \\
\hline Morbilidad posoperatoria, $n(\%)$ & $1(9.1)$ & $5(38.5)$ & $5(38.3)$ & $3(23.1)$ & 0.310 \\
\hline Tiempo quirúrgico $(\min )^{*}$ & $200 \pm 104$ & $207 \pm 101$ & $260 \pm 55$ & $224 \pm 103$ & 0.157 \\
\hline Hospitalización (días)* & $5.6 \pm 1.9$ & $6.2 \pm 1.6$ & $6.2 \pm 1.8$ & $9.1 \pm 4.3$ & 0.0470 \\
\hline Supervivencia $\left(\right.$ meses) ${ }^{*}$ & $74.0 \pm 49.6$ & $70.2 \pm 36.2$ & $19.3 \pm 10.2$ & $8.1 \pm 7.2$ & 0.0001 \\
\hline
\end{tabular}

*Media \pm desviación estándar.

de todos los casos durante un periodo mínimo de 12 meses (último caso reclutado), con excepción del fallecimiento de algún paciente.

\section{Aspectos éticos}

Se observaron las pautas éticas para la investigación en seres humanos definidas por la Declaración de Helsinki ${ }^{25}$.

\section{Resultados}

En el periodo analizado se realizaron 2329 colecistectomías por colelitiasis (agudas y crónicas) y se diagnosticaron 59 casos de CVB incidental, lo que representa una prevalencia en el periodo del $2.5 \%$. En ninguno de los casos hubo evidencias previas a la colecistectomía de la coexistencia de una neoplasia vesicular, y tampoco hubo casos de conversión a cirugía abierta ni de indicación de ampliación de cirugía durante la colecistectomía (en todos ellos fue tras el resultado del análisis de la pieza quirúrgica).

La distribución según el nivel de infiltración fue 9 casos (15.3\%) de pT1a, 11 casos (18.7\%) de pT1b, 13 casos $(22.0 \%)$ de pT2, 13 casos $(22.0 \%)$ de pT3 y 13 casos $(22.0 \%)$ de pT4. Tal como ya se ha comentado, los pacientes con pT1a fueron omitidos del análisis, por lo que se incluyeron 50 pacientes (pT1b, pT2, pT3 y pT4), con una media de edad de $58.6 \pm 9.6$ años (36-86 años), de los cuales 34 (68.0\%) eran mujeres (Tabla 1).

Cuando se analizaron los datos de laboratorio preoperatorios se observaron diferencias en los pacientes con pT4, respecto a los otros grupos, en las variables hematocrito, recuento total de leucocitos, fosfatasa alcalina, transaminasas y protrombina (Tabla 2).

En las imágenes tomográficas previas a la reintervención no se verificaron lesiones sospechosas de implantes secundarios en 48 pacientes (60.0\%).

La mediana del tiempo trascurrido entre la colecistectomía y la cirugía de resección hepática y linfadenectomía fue de 28 días (26 a 35 días).

Siguiendo el protocolo establecido, se realizó segmentectomía $s 4 b+s 5$ en 43 casos $(86.0 \%)$ y resección mayor en 7 casos (14.0\%): 4 hepatectomías derechas y 3 triseccionectomías derechas. En 22 casos (55.0\%) se halló infiltración del parénquima hepático y en 23 (57.5\%) en linfonodos regionales (Tabla 1); la mediana de linfonodos resecados fue de 7 (6 a 9). No se halló infiltración tumoral en el conducto cístico ni en ningún punto de acceso laparoscópico. El promedio del tiempo quirúrgico fue de $224 \pm 93$ minutos (100 a 450), y el promedio de la estancia hospitalaria fue de $6.9 \pm 2.9$ días (4 a 20).

En 43 pacientes $(86.0 \%)$ la resección fue $\mathrm{R} 0$, en 5 (10.0\%) fue R1 y en $2(4.0 \%)$ fue R2.

Con una mediana de seguimiento de 21 meses, observamos MPO asociada a la cirugía en 14 casos (28.0\%), de los que 8 son precoces (hasta 30 días): 6 pacientes desarrollaron atelectasia, que mejoró con ejercicios de respiración; 2 presentaron infección del sitio operatorio, que mejoró con curaciones; y en 6 aparecieron durante el seguimiento (hernia incisional). Por otra parte, se puede señalar que 8 casos correspondieron a Clavien-Dindo grado I (MPO precoces) y 6 a grado Illb (MPO de aparición tardía). No se registró mortalidad ni necesidad de reintervenciones. 
Tabla 2. Variables de laboratorio preoperatorias en relación con el grado de infiltración tumoral $(n=50)$

\begin{tabular}{lccccc}
\hline Variable & \multicolumn{3}{c}{ Nivel de infiltración tumoral } \\
\cline { 2 - 6 } & $\begin{array}{c}\mathbf{p} \text { T1b } \\
(\mathbf{n}=\mathbf{1 1})\end{array}$ & $\begin{array}{c}\mathbf{p T 2} \\
(\mathbf{n}=\mathbf{1 3})\end{array}$ & $\begin{array}{c}\mathbf{p T 3} \\
(\mathbf{n}=\mathbf{1 3})\end{array}$ & $\begin{array}{c}\mathbf{p T 4} \\
(\mathbf{n}=\mathbf{1 3})\end{array}$ & $\mathbf{p}$ \\
\hline Hematocrito (\%) & $37.7 \pm 1.3$ & $38.1 \pm 3.7$ & $38.3 \pm 1.7$ & $31.5 \pm 3.6$ & 0.0003 \\
Leucocitos totales (mm $)$ & $8472 \pm 929$ & $6384 \pm 1881$ & $8815 \pm 801$ & $11,838 \pm 2551$ & 0.0001 \\
Plaquetas (10 $)$ & $246 \pm 25$ & $224 \pm 59$ & $244 \pm 36$ & $274 \pm 12$ & 0.6016 \\
Bilirrubina total (mg/dl) & $0.8 \pm 0.2$ & $0.7 \pm 0.2$ & $0.9 \pm 0.2$ & $1.2 \pm 0.4$ & 0.0052 \\
Fosfatasa alcalina (U/l) & $166 \pm 49$ & $169 \pm 71$ & $209 \pm 55$ & $695 \pm 178$ & 0.0001 \\
ASAT (U/l) & $26.2 \pm 9.7$ & $22.3 \pm 6.8$ & $29.3 \pm 13.1$ & $121.8 \pm 60.4$ & 0.0001 \\
ALAT (U/l) & $25.2 \pm 8.9$ & $24.2 \pm 16.0$ & $29.5 \pm 16.7$ & $130.4 \pm 59.2$ & 0.0001 \\
Protrombina (\%) & $94.5 \pm 4.1$ & $93.4 \pm 3.7$ & $95.3 \pm 5.9$ & $77.6 \pm 13.1$ & 0.0011 \\
PTT (s) & $32.0 \pm 3.1$ & $30.4 \pm 3.1$ & $33.0 \pm 2.4$ & $37.8 \pm 5.6$ & 0.0027 \\
\hline
\end{tabular}

Los valores se expresan como media \pm desviación estándar.

En las alteraciones verificadas en los pacientes con tumores avanzados, el cáncer de vesícula biliar se asociaba a afecciones coexistentes (perforación tumoral del colon, infiltración en un quiste hidatídico hepático y coledocolitiasis residual).

ASAT: aspartato aminotransferasa; ALAT: alanino aminotransferasa; PTT: tiempo parcial de tromboplastina.

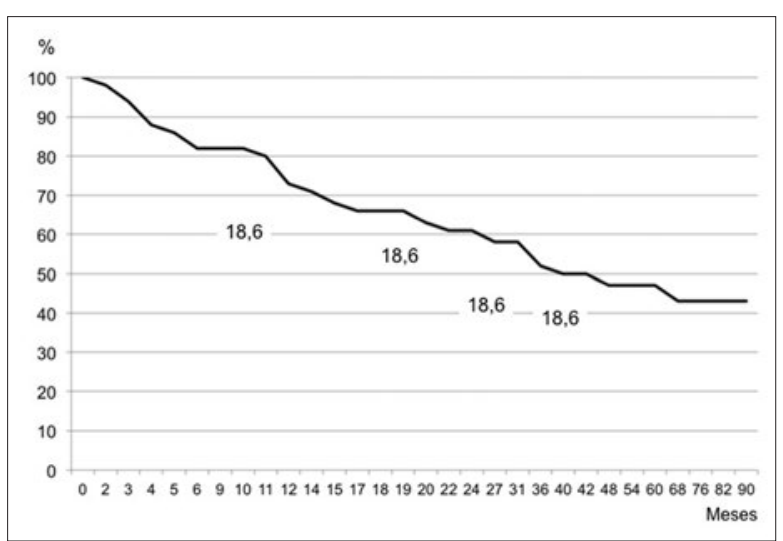

Figura 3. Supervivencia global actuarial de la serie, estimada mediante el método de Kaplan-Meier.

La adyuvancia realizada con una asociación de gemcitabina y 5-FU fue finalizada por 43 de los 50 pacientes (86.0\%); los siete restantes no recibieron o no completaron la adyuvancia (todos tenían tumores pT4, por lo que, en este subgrupo, la aplicabilidad del protocolo de quimioterapia fue del $46.0 \%$ ). Tres pacientes con pT4 y metástasis hepáticas no recibieron adyuvancia, y cuatro con pT4 fallecieron antes de completar el tratamiento complementario.

La SVG de la serie fue del 73.1, 61.0, 52.8, 49.9 y $47.0 \%$ a 1, 2, 3, 4 y 5 años, respectivamente (Fig. 3). La SVG por estadios se muestra en la figura 4.

\section{Discusión}

En esta serie de casos de CVB con tumores que superan la capa muscular (> pT1a) se demuestra la

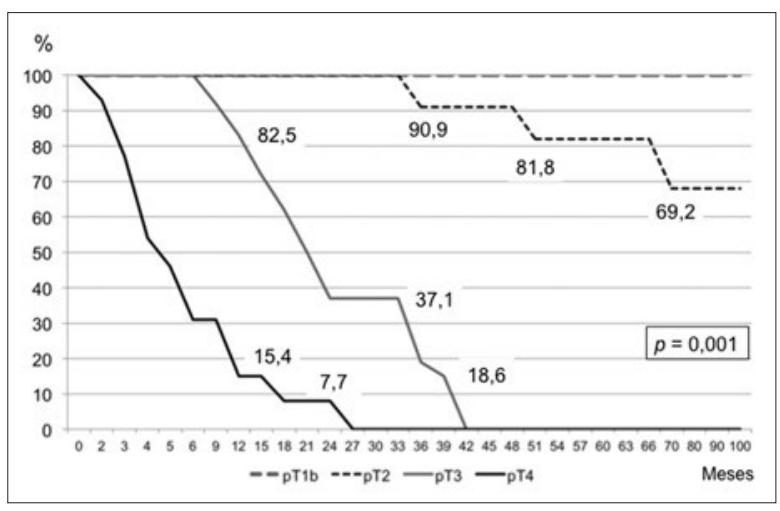

Figura 4. Supervivencia global actuarial según el nivel de invasión de la lesión principal, estimada mediante el método de Kaplan-Meier.

factibilidad de un protocolo de resección ampliada y tratamiento quimioterápico adyuvante en términos de morbimortalidad, obteniéndose una SVG a 5 años del $47.0 \%$. Sin embargo, estas conclusiones deben ser tomadas con cautela por las limitaciones inherentes del estudio. El número de casos incluido es reducido, y aunque el seguimiento ha sido estricto, se trata de una serie sin grupo de comparación. Pese a ello, hemos de hacer referencia a un estudio anterior de este mismo grupo de trabajo en el que se incluyeron CVB detectados tras colecistectomías abiertas y laparoscópicas, en los que se realizaron resecciones de menor calado y un esquema quimioterápico adyuvante distinto. Pese a esas diferencias, la SVG obtenida fue $\operatorname{similar}^{23}$. Con las limitaciones mencionadas no parece, pues, que una política generosa en la amplitud de la resección tenga impacto en la SVG. 
Un segundo hecho que cabe destacar es la utilidad de la clasificación basada en el nivel de infiltración de la pared de la vesícula biliar, 14,23. Por una parte, en este estudio, como en otros ${ }^{16,23}$, se demuestra una estricta correlación entre la supervivencia y el nivel de infiltración del CVB, y por otro, esta clasificación parece útil para decidir entre las opciones terapéuticas, máxime si se comprueba un $27.0 \%$ de pacientes con infiltración linfonodal en tumores $\mathrm{pT} 1 \mathrm{~b}$ y hasta un $15.0 \%$ de infiltración hepática en los pT2. Una parte fundamental del algoritmo es el escrupuloso estudio histopatológico de la pieza de colecistectomía, que incluye un «mapeo vesicular» (Fig. 2), y que tendrá luego implicaciones terapéuticas y pronósticas.

Finalmente, a tenor de nuestros resultados, no parece que en pacientes pT4, con invasión más allá de la serosa, la cirugía tenga un papel significativo en términos de prolongar la supervivencia, sino más bien como un procedimiento paliativo para mejorar la calidad de vida.

En cuanto a las características demográficas de nuestra serie, podemos decir que el comportamiento de la edad y el sexo no fue diferente a lo ya descrito en la literatura ${ }^{3,14,20,26,27}$ : hay un claro predominio del sexo femenino $(68.0 \%)$ y una edad media de presentación de $59.6 \pm 9.6$ años. Es de hacer notar que, cuando se analizó la edad en relación con el estadio tumoral, se verificó que en los pacientes con estadios avanzados esta fue algo menor (55.9 \pm 11.4 años), lo que permite sugerir el desarrollo de neoplasias más agresivas en las personas más jóvenes.

Desde el punto de vista quirúrgico, un aspecto crucial de un protocolo que contempla amplias resecciones quirúrgicas y linfadenectomías $D_{2}$, como el nuestro, es que no se registró mortalidad operatoria ni necesidad de reintervenciones. Los tiempos quirúrgicos, la MPO y la estancia hospitalaria fueron los esperables para este tipo de cirugía ${ }^{26,28-31}$.

Un punto sujeto a discusión es la magnitud de la resección hepática necesaria para cumplir criterios de radicalidad. Mientras algunos grupos sostienen que la simple resección de lecho hepático (margen de $2 \mathrm{~cm}$ de parénquima en el lecho) sería suficiente ${ }^{6,26,32}$, otros prefieren resecciones más amplias, como segmentectomías sIVb-sV o sIVb-sV-sVI, e incluso exéresis del colédoco $0^{9,20,33-35}$, y finalmente otros autores adoptan una posición intermedia, consistente en la resección de $2 \mathrm{~cm}$ de parénquima en el lecho junto con resección del colédoco supraduodenal ${ }^{3,13}$. En el protocolo de actuación de la serie actual se optó por resecciones amplias que, si bien tuvieron una MPO aceptable, no parecieron proporcionar una supervivencia mayor si se compara con la obtenida en una serie anterior ( $50 \%$ a 5 años) en la que su utilizaron criterios menos rígidos ${ }^{23}$. Otros autores tampoco parecen haber relacionado la magnitud de la resección hepática y la supervivencia ${ }^{36}$, pero queda todavía pendiente la respuesta a algunas controversias en este campo, como la indicación de colecistectomía extendida en tumores pT1a y pT1b, y el papel que los senos de Rokitansky-Aschoff pudieran tener en la supervivencia ${ }^{10}$. En cuanto al primer punto, hay autores que han demostrado supervivencias del 87.6 y el $86.4 \%$ en pacientes con pT1a y pT1b, respectivamente, al ser tratados con colecistectomía exclusiva, con una caída de 30 puntos porcentuales en aquellos con invasión de los senos de Rokitansky-Aschoff ${ }^{10}$.

La reintervención tiene dos objetivos concretos: lograr una resección $\mathrm{R} 0$ y la exéresis de los linfonodos, permitiendo además una correcta estadificación de los pacientes ${ }^{37}$. Por otra parte, se considera que una linfadenectomía adecuada incluye la valoración de cualquier linfonodo regional sospechoso, de los linfonodos interaortocava y la recuperación de al menos seis linfonodos, lo que permite lograr una apropiada estadificación del paciente ${ }^{38}$. Por ende, desde el punto de vista de la radicalidad de la cirugía, las evidencias sustentan la realización de una linfadenectomía $D_{2}$, no solo por su pretensión curativa, sino también por permitir una mejor estadificación ${ }^{16,28,39}$.

En el $46.2 \%$ (6/13) de los pacientes T3 y el $100 \%$ (13/13) de los T4 se evidenció infiltración parenquimatosa de la neoplasia (Tabla 1), pero en ninguno de ellos a la vía biliar. De hecho, en ningún caso se constató infiltración tumoral microscópica del conducto cístico, lo que puede explicar los bajos valores de bilirrubina en ambos subgrupos y las cifras elevadas de fosfatasa alcalina en los T4 (Tabla 2).

Otro dato que pudiera tener interés es el posible implante de células tumorales en los puertos de acceso de la colecistectomía en aquellos pacientes en los que posteriormente se detecta un CVB. Tal como se ha descrito en la metodología, y siguiendo las recomendaciones de otros autores ${ }^{9,34,35,40-42}$, se realizó exéresis de las heridas quirúrgicas laparoscópicas en todos los pacientes. Sin embargo, no se detectó actividad tumoral en ninguno de ellos, por lo que parece que esta recomendación, pese a su base teórica y algunas evidencias, solo tendría sentido si los estudios por imagen detectan alguna alteración, pero no sistemáticamente. Al respecto, se podría comentar, además, que el uso de una bolsa plástica para el 
retiro del espécimen (según protocolo para nosotros) pudo haber influido en el hecho de no registrar implantes tumorales en los puertos de acceso.

La utilización de quimioterapia adyuvante sigue siendo motivo de controversia ${ }^{18,43,44}$. Aunque algunos autores sugieren su administración en pacientes con pT2, pT3 y pT4, con infiltración linfonodal o con márgenes comprometidos, no existe consenso respecto al esquema que debe utilizarse ${ }^{45-54}$. Nosotros optamos por una asociación de gemcitabina y 5-FU. Aunque existe evidencia del incremento de la supervivencia, a partir de un estudio de fase III en el que se asoció gemcitabina, oxaliplatino y erlotinib55, y de otro de fase II con gemcitabina, cisplatino, oxaliplatino y 5-FU ${ }^{54}$, nuestros resultados no parecen dar soporte a un incremento de la supervivencia utilizando adyuvancia. En este sentido, quizás haya que apuntar hacia los resultados obtenidos con estudios genéticos del CVB, en los que destaca la aparición de mutaciones de los oncogenes KRAS (19.0 a 38.0\%) y BRAF (33.0\%), y de los genes supresores TP53 (36.0\%) y P16INK4A (31.0 a 62.0\%), lo que sugiere la posibilidad de tratamientos personalizados ${ }^{56-58}$.

La SVG de la serie, del $47.0 \%$ a 60 meses, es similar a la descrita en otros trabajos ${ }^{13}$, pero si nos atenemos a la SVG por estadios pudo verificarse que los pacientes con estadios pT3 y pT4 tienen una SVG a 12 y 24 meses del 82.5 y el $37.1 \%$, y del 15.4 y el $7.7 \%$, respectivamente, lo que es inferior a lo observado en una serie china, en la que se reportó un $44.4 \%$ de SVG a 5 años para pacientes pT $^{9}$.

\section{Conclusión}

Podemos señalar que nuestros resultados, con las limitaciones apuntadas, demuestran la factibilidad de un protocolo de resección ampliada y de quimioterapia adyuvante en el CVB que supera la capa muscular, con tasas de MPO y mortalidad adecuadas, y SVG similar a la reportada anteriormente.

\section{Conflicto de intereses}

Los autores declaran que no tienen ningún conflicto de intereses para el desarrollo y la publicación del trabajo.

\section{Financiamiento}

Financiado parcialmente por Proyecto MEC 80170022 , CONICYT.

\section{Responsabilidades éticas}

Protección de personas y animales. Los autores declaran que para esta investigación no se han realizado experimentos en seres humanos ni en animales.

Confidencialidad de los datos. Los autores declaran que han seguido los protocolos de su centro de trabajo sobre la publicación de datos de pacientes.

Derecho a la privacidad y consentimiento informado. Los autores declaran que en este artículo aparecen datos de pacientes, obtenidos a partir de sus historiales.

\section{Bibliografía}

1. Itriago L, Silva N, Cortés G. Cáncer en Chile y el mundo: una mirada epidemiológica, presente y futuro. Rev Med Clin Condes. 2013;24:531-52.

2. American Cancer Society. Estadísticas de supervivencia para el cáncer de vesícula biliar, según la etapa. (Consultado el 31 de enero de 2018.) Disponible en: https://www.cancer.org/es/cancer/cancer-de-vesicula-biliar/ deteccion-diagnostico-clasificacion-por-etapas/tasas-de-supervivencia.htm

3. He XD, Li JJ, Liu W, Qu Q, Hong T, Xu XQ, et al. Surgical procedure determination based on tumor-node-metastasis staging of gallbladder cancer. World J Gastroenterol. 2015;21:4620-6.

4. Maluenda F, Díaz JC, Aretxabala X, Burdiles P, Csendes A, Contreras L. Strategies for the surgical treatment of gallbladder cáncer. Rev Med Chil. 2005;133:723-8.

5. Roa I, Ibacache G, Muñoz S, de Aretxabala X. Gallbladder cancer in Chile: pathologic characteristics of survival and prognostic factors: analysis of 1,366 cases. Am J Clin Pathol. 2014;141:675-82.

6. Lee W, Jeong CY, Jang JY, Kim YH, Roh YH, Kim KW, et al. Do hepatic-sided tumors require more extensive resection than peritoneal-sided tumors in patients with T2 gallbladder cancer? Results of a retrospective multicenter study. Surgery. 2017;162:515-24.

7. Shindoh J, de Aretxabala X, Aloia TA, Roa JC, Roa I, Zimmitti G, et al. Tumor location is a strong predictor of tumor progression and survival in t2 gallbladder cancer: an international multicenter study. Ann Surg. 2015;261:733-9.

8. Ahn Y, Park CS, Hwang S, Jang HJ, Choi KM, Lee SG. Incidental gallbladder cancer after routine cholecystectomy: when should we suspect it preoperatively and what are predictors of patient survival? Ann Surg Treat Res. 2016:90:131-8.

9. Tian YH, Ji X, Liu B, Yang GY, Meng XF, Xia HT, et al. Surgical treatment of incidental gallbladder cancer discovered during or following laparoscopic cholecystectomy. World J Surg. 2015;39:746-52.

10. Roa JC, Tapia O, Manterola C, Villaseca M, Guzmán P, Araya JC, et al Early gallbladder carcinoma has a favorable outcome but Rokitansky-Aschoff sinus involvement is an adverse prognostic factor. Virchows Arch. 2013:463:651-61.

11. Ethun CG, Postlewait LM, Le N, Pawlik TM, Buettner S, Poultsides G, et al. A novel pathology-based preoperative risk score to predict locoregional residual and distant disease and survival for incidental gallbladder cancer: a 10-institution study from the U.S. Extrahepatic Biliary Malignancy Consortium. Ann Surg Oncol. 2017;24:1343-50.

12. Piccolo G, Di Vita M, Cavallaro A, Fisichella R, Zanghì $A$, Spartà $D$, et al. Lymph node evaluation in gallbladder cancer: which role in the prognostic and therapeutic aspects. Update of the literature. Eur Rev Med Pharmacol Sci. 2014;18(2 Suppl):47-53.

13. Shirai $Y$, Wakai $T$, Sakata J, Hatakeyama K. Regional lymphadenectomy for gallbladder cancer: rational extent, technical details, and patient outcomes. World J Gastroenterol. 2012;18:2775-83.

14. Muratore A, Amisano M, Viganò L, Massucco P, Capussotti L. Gallbladder cancer invading the perimuscular connective tissue: results of reresection after prior non-curative operation. J Surg Oncol. 2003;83:212-5.

15. Sikora SS, Singh RK. Surgical strategies in patients with gallbladder cancer: nihilism to optimism. J Surg Oncol. 2006;93:670-81.

16. Sternby Eilard M, Lundgren L, Cahlin C, Strandell A, Svanberg T, Sandström P. Surgical treatment for gallbladder cancer - a systematic literature review. Scand J Gastroenterol. 2017:52:505-14.

17. Shirai Y, Wakai T, Hatakeyama K. Radical lymph node dissection for gallbladder cancer: indications and limitations. Surg Oncol Clin N Am. 2007;16:221-32. 
18. de Aretxabala X, Roa I, Berrios M, Hepp J, Gallardo J, Cordova A, et al. Chemoradiotherapy in gallbladder cancer. J Surg Oncol. 2006;93:699-704

19. Foster JM, Hoshi H, Gibbs JF, Iyer R, Javle M, Chu Q, et al. Gallbladder cancer: Defining the indications for primary radical resection and radica re-resection. Ann Surg Oncol. 2007;14:833-40.

20. Yamaguchi J, Kaneoka Y, Maeda A, Takayama Y, Onoe S, Isogai M. Benefit of extended radical surgery for incidental gallbladder carcinoma. Surg Today. 2016;46:453-9.

21. Manterola $\mathrm{C}$, Otzen $\mathrm{T}$. Checklist for reporting results using observational descriptive studies as research designs. The MInCir Initiative. Int J Morphol. 2017;35:72-6.

22. Clavien PA, Barkun J, de Oliveira ML, Vauthey JN, Dindo D, Schulick RD, et al. The Clavien-Dindo classification of surgical complications: five-year experience. Ann Surg. 2009;250:187-96.

23. Manterola C, Vial M, Roa JC. Survival of a cohort of patients with intermediate and advanced gall bladder cancer treated with a prospective therapeutic protocol. Acta Cir Bras. 2010;25:225-30.

24. Manterola C, Conejeros R, Yáñez E. ¿Cuál es el rol de la cirugía en el tratamiento de un paciente con cáncer de la vesícula biliar estadio Illb? Int J Morphol. 2015;33:653-9.

25. Helsinki Statement of the Worldwide Medical Association. Ethical principles for the medical researches in human beings. Amended by $52 a$ General Assembly, Edinburgh, Scotland; October 2000.

26. Goetze TO. Gallbladder carcinoma: prognostic factors and therapeutic options. World J Gastroenterol. 2015; 21:12211-7.

27. Wang JD, Liu YB, Quan ZW, Li SG, Wang XF, Shen J. Role of regional lymphadenectomy in different stage of gallbladder carcinoma. Hepatogastroenterology. 2009;56:593-6.

28. Birnbaum DJ, Viganò L, Russolillo N, Langella S, Ferrero A, Capussotti L. Lymph node metastases in patients undergoing surgery for a gallbladder cancer. Extension of the lymph node dissection and prognostic value of the lymph node ratio. Ann Surg Oncol. 2015;22:811-8.

29. Giuliante F, Ardito F, Vellone M, Clemente G, Nuzzo G. Port-sites excision for gallbladder cancer incidentally found after laparoscopic cholecystectomy. Am J Surg. 2006;191:114-6.

30. Hamila F, Letaief R, Khnissi M, Derbel F, Mazhoud J, Ben Ali A et al. Port site recurrence after laparoscopic cholecystectomy. Tunis Med. 2006;84:697-700.

31. Paolucci V. Port site recurrences after laparoscopic cholecystectomy. J Hepatobiliary Pancreat Surg. 2001;8:535-43.

32. Goetze TO, Paolucci V. Incidental T1b-T3 gallbladder carcinoma. Extended cholecystectomy as an underestimated prognostic factor-results of the German registry. Chirurg. 2014;85:131-8.

33. Choi SB, Han HJ, Kim WB, Song TJ, Suh SO, Choi SY. Surgical strategy for T2 and T3 gallbladder cancer: is extrahepatic bile duct resection always necessary? Langenbecks Arch Surg. 2013:398:1137-44.

34. Kawahara R, Shirahama T, Arai S, Muroya D, Nomura Y, Fukutomi S, et al. Evaluation of surgical procedures for T2 gallbladder cancer in terms of recurrence and prognosis. Kurume Med J. 2017;63:15-22.

35. Utsumi M, Aoki H, Kunitomo T, Mushiake Y, Yasuhara I, Arata T, et al Evaluation of surgical treatment for incidental gallbladder carcinoma diagnosed during or after laparoscopic cholecystectomy: single center results. BMC Res Notes. 2017;10:56

36. Lee SE, Kim KS, Kim WB, Kim IG, Nah YW, Ryu DH, et al.; Korean Association of Hepato-Biliary and Pancreas Surgery. Practical guidelines for the surgical treatment of gallbladder cancer. J Korean Med Sci. 2014;29:1333-40.

37. Cavallaro A, Piccolo G, Di Vita M, Zanghì A, Cardì F, Di Mattia $P$, et al Managing the incidentally detected gallbladder cancer: algorithms and controversies. Int J Surg. 2014;12(Suppl 2):S108-19.

38. Aloia TA, Járufe N, Javle M, Maithel SK, Roa JC, Adsay V, et al. Gallbladder cancer: expert consensus statement. HPB (Oxford). 2015;17:681-90.

39. Shroff RT, Knox J, Dixon E. Consensus conference on gallbladder cancer. HPB (Oxford). 2015;17:664-5.
40. Liu Q, Wu N, Zhou N. Port-site resection in surgical management of incidental gallbladder cancer: a still unresolved question. Ann Surg Oncol. 2017;24(Suppl 3):646.

41. Jang JY, Heo JS, Han Y, Chang J, Kim JR, Kim H, et al. Impact of type of surgery on survival outcome in patients with early gallbladder cancer in the era of minimally invasive surgery: oncologic safety of laparoscopic surgery. Medicine (Balt.). 2016;95:e3675.

42. Sheikh MR, Osman H, Cheek S, Hunter S, Jeyarajah DR. T2 Gallbladder cancer-aggressive therapy is warranted. Am Surg. 2016;82:518-21.

43. de Aretxabala X, Roa I, Burgos L. Gallbladder cancer, management of early tumors. Hepatogastroenterology. 1999:46:1547-51.

44. Lee J, Park SH, Chang HM, Kim JS, Choi HJ, Lee MA, et al. Gemcitabine and oxaliplatin with or without erlotinib in advanced biliary-tract cancer: a multicentre, open-label, randomised, phase 3 study. Lancet Oncol. 2012;13:181-8.

45. Chen L, Chen C, Yen Y, Tam KW. Chemotherapy for advanced biliary tract carcinoma: a meta-analysis of randomized controlled trials. Medicine (Balt.). 2016;95:e4584.

46. Creasy JM, Goldman DA, Dudeja V, Lowery MA, Cercek A, Balachandran VP, et al. Systemic chemotherapy combined with resection for locally advanced gallbladder carcinoma: surgical and survival outcomes. J Am Coll Surg. 2017;224:906-16.

47. Eckel F, Schmid RM. Chemotherapy and targeted therapy in advanced biliary tract carcinoma: a pooled analysis of clinical trials. Chemotherapy. 2014;60:13-23.

48. Lee KJ, Yi SW, Cha J, Seong J, Bang S, Song SY, et al. A pilot study of concurrent chemoradiotherapy with gemcitabine and cisplatin in patients with locally advanced biliary tract cancer. Cancer Chemother Pharmacol. 2016;78:841-6.

49. Mantripragada KC, Hamid F, Shafqat H, Olszewski AJ. Adjuvant therapy for resected gallbladder cancer: analysis of the National Cancer Data Base. J Natl Cancer Inst. 2016;109(2). pii: djw202.

50. Nakamura M, Nakashima $H$, Abe $T$, Ensako $T$, Yoshida $K$, Hino $K$ Gemcitabine-based adjuvant chemotherapy for patients with advanced gallbladder cancer. Anticancer Res. 2014;34:3125-9.

51. Neoptolemos JP, Moore MJ, Cox TF, Valle JW, Palmer DH, McDonald AC, et al. Effect of adjuvant chemotherapy with fluorouracil plus folinic acid or gemcitabine vs observation on survival in patients with resected periampullary adenocarcinoma: the ESPAC-3 periampullary cancer randomized trial. JAMA. 2012;308:147-56.

52. Sohn BS, Yuh YJ, Kim KH, Jeon TJ, Kim NS, Kim SR. Phase II trial of combination chemotherapy with gemcitabine, 5-fluorouracil and cisplatin for advanced cancers of the bile duct, gallbladder, and ampulla of Vater. Tumori. 2013;99:139-44.

53. Wang SJ, Lemieux A, Kalpathy-Cramer J, Ord CB, Walker GV, Fuller CD, et al. Nomogram for predicting the benefit of adjuvant chemoradiotherapy for resected gallbladder cancer. J Clin Oncol. 2011;29:4627-32.

54. Yamashita Y, Taketomi A, Fukuzawa K, Yoshizumi T, Uchiyama H, Simada $\mathrm{M}$, et al. Gemcitabine combined with 5 -fluorouracil and cisplatin (GFP) in patients with advanced biliary tree cancers: a pilot study. Anticancer Res. 2006;26:771-5.

55. Yang R, Wang B, Chen YJ, Li HB, Hu JB, Zou SQ. Efficacy of gemcitabine plus platinum agents for biliary tract cancers: a meta-analysis. Anticancer Drugs. 2013;24:871-7.

56. Hezel AF, Deshpande V, Zhu AX. Genetics of biliary tract cancers and emerging targeted therapies. J Clin Oncol. 2010;28:3531-40.

57. Marino D, Leone F, Cavalloni G, Cagnazzo C, Aglietta M. Biliary tract carcinomas: from chemotherapy to targeted therapy. Crit Rev Oncol Hematol. 2013;85:136-48.

58. Zhu AX, Hezel AF. Development of molecularly targeted therapies in biliary tract cancers: reassessing the challenges and opportunities. Hepatology. 2011;53:695-704 